\title{
Opioid Prescribing by Primary Care Providers: a Cross-Sectional Analysis of Nurse Practitioner, Physician Assistant, and Physician Prescribing Patterns
}

\author{
M. James Lozada, DO', Mukaila A. Raji, MD2,3,4, James S. Goodwin, MD ${ }^{2,3,4}$, and \\ Yong-Fang Kuo, $\mathrm{PhD}^{2,3,4}$
}

\begin{abstract}
'Department of Anesthesiology, Vanderbilt University Medical Center, 1211 Medical Center Dr., 4202 VUH, Nashville, TN, USA; ${ }^{2}$ Department of Internal Medicine and Sealy Center on Aging, University of Texas Medical Branch, Galveston, TX, USA; ${ }^{3}$ Department of Preventive Medicine and Community Health, University of Texas Medical Branch, Galveston, TX, USA; ${ }^{4}$ Institute for Translational Science, University of Texas Medical Branch, Galveston, TX, USA
\end{abstract}

BACKGROUND: Prescription opioid overprescribing is a focal point for legislators, but little is known about opioid prescribing patterns of primary care nurse practitioners (NPs) and physician assistants (PAs).

OBJECTIVE: To identify prescription opioid overprescribers by comparing prescribing patterns of primary care physicians (MDs), nurse practitioners (NPs), and physician assistants (PAs).

DESIGN: Retrospective, cross-sectional analysis of Medicare Part D enrollee prescription data.

PARTICIPANTS: Twenty percent national sample of 2015 Medicare Part D enrollees.

MAIN MEASURES: We identified potential opioid overprescribing as providers who met at least one of the following: (1) prescribed any opioid to $>50 \%$ of patients, (2) prescribed $\geq 100$ morphine milligram equivalents (MME)/ day to $>10 \%$ of patients, or (3) prescribed an opioid $>$ 90 days to $>20 \%$ of patients.

KEY RESULTS: Among 222,689 primary care providers, $3.8 \%$ of MDs, $8.0 \%$ of NPs, and $9.8 \%$ of PAs met at least one definition of overprescribing. $1.3 \%$ of $\mathrm{MDs}, 6.3 \%$ of NPs, and $8.8 \%$ of PAs prescribed an opioid to at least $50 \%$ of patients. NPs/PAs practicing in states with independent prescription authority were $>20$ times more likely to overprescribe opioids than NPs/PAs in prescriptionrestricted states.

CONCLUSIONS: Most NPs/PAs prescribed opioids in a pattern similar to MDs, but NPs/PAs had more outliers who prescribed high-frequency, high-dose opioids than did MDs. Efforts to reduce opioid overprescribing should include targeted provider education, risk stratification, and state legislation.

KEY WORDS: opioid prescribing; primary care providers; nurse practitioner; physician assistant; opioid epidemic.

Prior presentation: None.

Electronic supplementary material The online version of this article (https://doi.org/10.1007/s11606-020-05823-0) contains supplementary material, which is available to authorized users.

Received November 3, 2019

Accepted March 26, 2020

Published online April 24, 2020
J Gen Intern Med 35(9):2584-92

DOI: $10.1007 / \mathrm{s} 11606-020-05823-0$

(C) Society of General Internal Medicine 2020

\section{INTRODUCTION}

Americans aged 65 and older receive a disproportionately large number of prescription opioids. ${ }^{1}$ In 2018 , nearly onethird of 47 million Medicare Part D beneficiaries received at least one opioid prescription. ${ }^{2}$ Paralleling this number is the increased incidence of opioid-related overdose and death, ${ }^{3}$ especially in the older population. ${ }^{4,5}$ Among those admitted to hospitals for prescription opioid overdose, Medicare is the most common patient insurance status. ${ }^{6}$ The toxicity from opioids has led to state policies and federal guidelines aimed to curb opioid overprescribing. ${ }^{7-10}$ According to the Centers for Disease Control and Prevention (CDC) guideline for prescribing opioids for non-cancer pain, "Clinicians should avoid increasing dosage to $\geq 90$ morphine milligram equivalents (MME)/day or carefully justify a decision to titrate dosage to $\geq 90 \mathrm{MME} /$ day. For acute pain, three days or less of opioids will often be sufficient; more than seven days will rarely be needed." "11 Despite CDC guidelines, in 2018, more than 350,000 Medicare Part D enrollees without a cancer diagnosis received opioid prescriptions averaging $>120 \mathrm{MME} /$ day for at least 3 months. ${ }^{2}$

A key step to reduce opioid overprescribing is to examine prescribing profiles of both physician and non-physician prescribers. Prior studies have focused on understanding physician (MD) overprescribing and the impact of federal opioid policy. However, information is limited about opioid prescribing by nurse practitioners (NPs) and physician assistants (PAs), the fastest growing segment of the primary care provider population. ${ }^{12-14} \mathrm{NPs}$ in many states are allowed to independently prescribe schedule II opioids without physician oversight, ${ }^{15-17}$ and the training, licensure standards, and continuous medical education requirements for NPs/PAs vary between states. Finally, MD and non-MD prescribers differ in medical training and recertification requirements. 
Given these differences, one might expect differences in opioid prescribing practices between MD and non-MD prescribers.

We conducted a retrospective, cross-sectional analysis of 2015 Medicare claims data to characterize primary care provider opioid prescribing by analyzing patient- and providerlevel data. We hypothesized that NPs and PAs would prescribe fewer opioids with a lower MME/day than MDs. We further hypothesized that NPs/PAs in states without independent authority to prescribe schedule II opioids would prescribe opioids less frequently, with a lower MME/day, and for shorter periods than those in states with independent prescribing authority.

\section{METHODS}

\section{Overview}

We gathered opioid prescribing data of primary care MDs, NPs, and PAs and reported each group's opioid prescribing rate. We then examined individual providers to identify potential opioid overprescribers.

\section{Data Source}

This study followed the Strengthening the Reporting of Observational Studies in Epidemiology (STROBE) guidelines for reporting cross-sectional studies. ${ }^{18}$ We used enrollment and claims data from a randomly selected, $20 \%$ national sample of Medicare beneficiaries enrolled in 2015. The dataset included Medicare beneficiary summary files, Outpatient Standard Analytic Files (OUTSAF), Medicare Carrier files, and Prescription Drug Event (PDE) files. The University of Texas Medical Branch Institutional Review Board approved the research.

\section{Study Cohorts}

We included providers from the Medicare Part D file who prescribed any medication. To identify provider specialties, we used Part B claims in the Medicare Carrier file. Providers with multiple specialty codes were assigned the specialty that appeared most often in their 2015 claims. We categorized providers as primary care MDs, primary care NPs, and primary care PAs (Supplemental Table 1). NPs/PAs working in specialty fields were identified by Healthcare Provider Taxonomy Codes (such as surgery, critical care, acute care, obstetrics and gynecology, psychiatric and mental health, or occupational health) then excluded from the primary care NP/PA cohort. We obtained taxonomy codes by linking National Provider Identifier (NPI) numbers from the Medicare Part D file to the National Plan and Provider Enumeration System (NPPES) file available at the CMS website.

We selected primary care providers who prescribed at least 50 prescriptions. To exclude providers practicing in emergency settings, we chose providers who billed $\leq 50 \%$ of E\&M services for emergency department visits. We also excluded providers who practiced outside the 50 United States and Washington D.C. The steps for generating each cohort are included in Supplemental Table 2.

\section{Opioid Prescribing}

We identified opioid prescriptions in the 2015 Medicare Part D file using definitions from the National Drug Code, product name, therapeutic class description, and DEA class code from the 2015 RedBook Select Extracts database. Prescriptions for methadone were included in analysis; buprenorphine, naltrexone, and injection opioids were excluded.

For each provider, we identified all patients who received any medication, then calculated the percent who received any opioid, a high-dose opioid, or a long-term opioid prescription. A high-dose opioid prescription was defined as containing $\geq 100 \mathrm{MME} /$ day. A long-term opioid prescription was defined as $>90$-day opioid supply in 2015. In these analyses, a patient could be associated with multiple primary care providers, provided they prescribed at least one medication to the patient.

\section{Potential Overprescriber Definition}

We defined potential overprescribers as providers who met at least one of the following: (1) prescribed any opioid to $>50 \%$ of patients (high frequency), (2) prescribed $\geq 100 \mathrm{MME} /$ day to $>10 \%$ of patients (high dose), or (3) prescribed an opioid > 90 days to $>20 \%$ of patients (long term). The cutoffs chosen were about two standard deviations higher than the mean for all primary care providers.

\section{Patient Characteristics}

Medicare enrollment files indicated patient age, gender, race/ethnicity, and original entitlement. We used a Medicaid indicator in the enrollment file as a proxy for low income. We categorized residential areas into metropolitan, nonmetropolitan urban, and rural using Rural-Urban Continuum Codes. ${ }^{19}$

\section{Provider Characteristics}

To characterize each provider's unique group of patients, we calculated the percent of patients $\geq 80$ years, female, minority, Medicaid eligible, disability as the original entitlement for Medicare, and HMO enrollment from the patient data of each provider. We also obtained rurality and primary care physician availability per 10,000 population, by the county of practice. ${ }^{20}$

\section{State Law on Schedule II Prescribing by NPs/ PAs}

We further categorized the practice location of NPs/PAs into states which either allowed them to independently prescribe schedule II opioids or states which required physician oversight for NP/PA opioid prescribing. In 2015, 8 states restricted 
NP independent prescription authority: Alabama, Arkansas, Florida, Georgia, Missouri, Oklahoma, South Carolina, and West Virginia. ${ }^{21}$ Eight states also restricted PA independent prescription authority in 2015: Alabama, Arkansas, Florida, Georgia, Iowa, Kentucky, Missouri, and West Virginia. ${ }^{22,} 23$ Most of these states prevented NP/PA prescribing of schedule II controlled substances, even with physician oversight (Supplemental Table 3 provides prescriptive authority details). To show state-level differences in NP/PA opioid prescribing, we calculated the mean MME prescribed by NPs/PAs to each Part $\mathrm{D}$ enrollee. This was calculated as the total MME prescribed by NPs/PAs divided by the total number of Part $D$ enrollees in each state. We also reported the percentage of opioids prescribed by primary care NPs/PAs compared with primary care MDs. These calculations included all primary care providers without limitations on a providers' patient load or number of prescriptions.

\section{Statistical Analyses}

Histograms were plotted which detailed the percent of patients that received any opioid, high-dose opioids, and/ or long-term opioids, by individual primary care providers. Three conditional logistic regression models were applied to identify provider characteristics associated with each definition of potential overprescribing within each state. Analyses were performed with SAS Enterprise version 7.12 at the CMS Virtue Research Data Center (SAS Inc., Cary, NC). Maps were constructed using ArcGIS 9.3 (Esri, Redlands, CA).

\section{Sensitivity Analysis}

To control for differences in patient comorbidity, we repeated our analyses on a subset of primary care providers. We identified comorbidity for enrollees with complete data, based on Part A and B coverage and no HMO enrollment in 2014. We then determined each patient's number of comorbidities. Finally, we added the percentage of patients with $\geq 3$ comorbidities, based on the Elixhauser comorbidity index, as a provider characteristic into the conditional logistic regression models. The Elixhauser comorbidity index includes 30 physical or mental conditions, many of which are associated with opioid prescribing. ${ }^{24}$

Next, we sought to verify the robustness of our classification of NPs/PAs as primary care providers. Using social network analysis, we identified primary care practices (Supplemental Table 4). We then repeated our analyses among MDs, NPs, and PAs, stratified by whether or not they belonged to a primary care practice. ${ }^{25-27}$

\section{RESULTS}

The 20\% national sample of the 2015 Medicare Part D file contained 449,194 NPI numbers (primary care MD 244,862;
NP 121,949; PA 82,383). Study cohorts were created following pre-specified exclusion criteria leaving 156,161 MDs; 42,655 NPs; and 23,873 PAs for analysis (Supplemental Table 2).

\section{Potential Opioid Overprescribing by Primary Care MDs, NPs, and PAs}

Table 1 and Figure 1a show just 1.33\% of primary care MDs $(n=2079)$ were high-frequency opioid prescribers (any opioid to $>50 \%$ of patients) compared with $6.34 \%$ of NPs $(n=2704)$ and $8.84 \%$ of PAs $(n=2111)$. Figure $1 \mathrm{~b}$ demonstrates that most primary care providers did not prescribe any high-dose opioids ( $\geq 100 \mathrm{MME} /$ day) in 2015 (MD 72.6\%, NP 82.5\%, PA 82.3\%). However, as seen in Table 1 and Figure 1b, a small percentage of primary care providers prescribed highdose opioids to more than $10 \%$ of their patients (MD $0.95 \%$, NP $2.89 \%$, PA $3.41 \%$ ). Table 1 and Figure 1c show that, while fewer NPs/PAs prescribed long-term opioids ( $>90$ days/year) than MDs, more NPs/PAs prescribed long-term opioids to more than $20 \%$ of their patients than MDs (MD 2.93\%, NP $3.90 \%$, PA $3.65 \%$ ).

In multivariable analyses (Table 2), NPs/PAs were more likely than MDs to prescribe high-frequency (OR: NP, 2.96; PA, 5.73) and high-dose opioids (OR: NP, 1.66; PA, 2.16); but NPs/PAs were less likely than MDs to prescribe long-term opioids (OR: NP, 0.57; PA, 0.71). Among all providers, each definition of overprescribing was associated with providers who had a higher proportion of patients older than age 80 , female, White, or disabled. Providers who practiced in urban areas were more likely to prescribe high-frequency and high-dose opioids but were less likely to prescribe long-term opioids (Table 2).

\section{Association of Opioid Prescribing with State Prescription Authority Laws}

Figure 2 shows that the amount of opioids prescribed by primary care NPs/PAs varied significantly across the country. Among states in the lowest quintile of opioid prescribing, most had laws in 2015 which restricted NP/ PA authority to independently prescribe schedule II opioids (exceptions are Hawaii, Louisiana, and Texas). In states which allowed independent prescription authority, NPs/PAs were more likely to prescribe high-frequency opioids than NPs/PAs in states which restricted authority (NP, $7.5 \%$ vs $0.2 \%$; PA, $10.0 \%$ vs $0.7 \%$; Table 1 ). In multivariable analysis, NPs/PAs who practiced in states with independent prescription authority were about 28 times more likely to overprescribe opioids than NPs/PAs in states which restricted authority (OR, 28.2; 95\% CI, 19.67-40.44). These results were even more striking for high-dose (OR, 70.1; 95\% CI, 29.13-168.61) and longterm (OR, 46.9; 95\% CI, 25.84-84.99) definitions of overprescribing (Supplemental Table 5). 


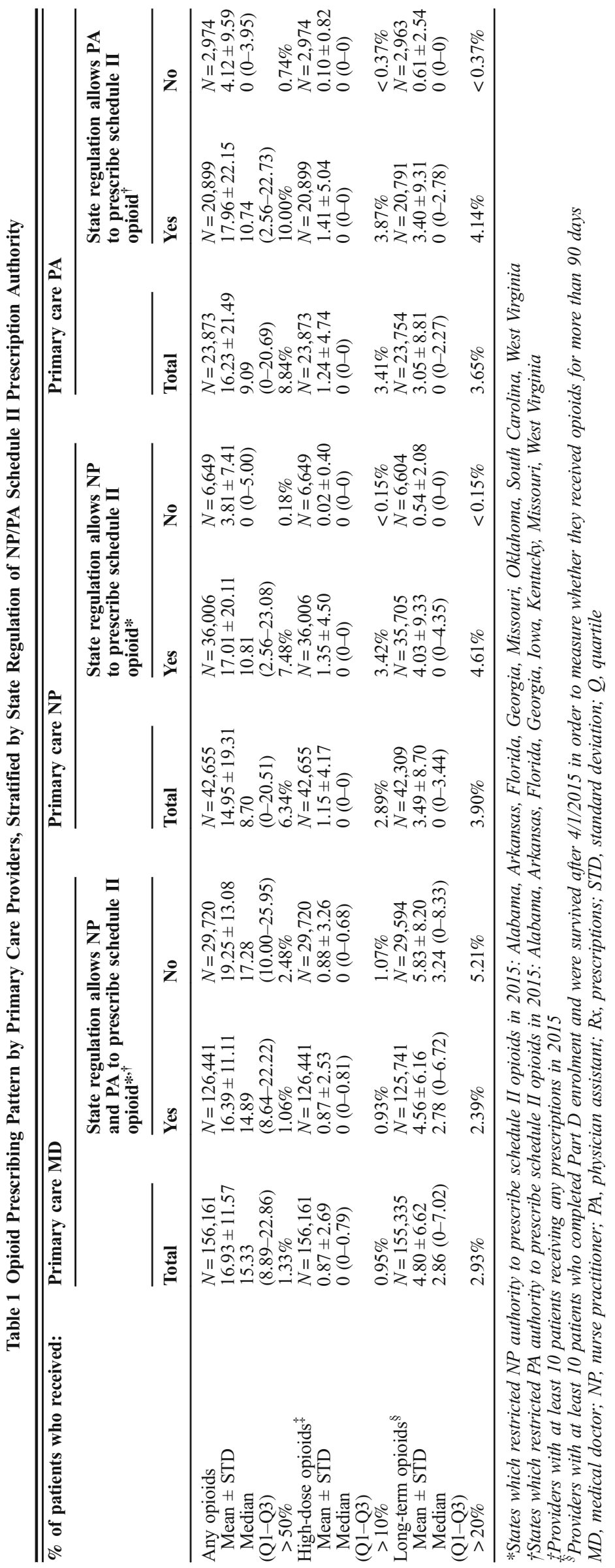



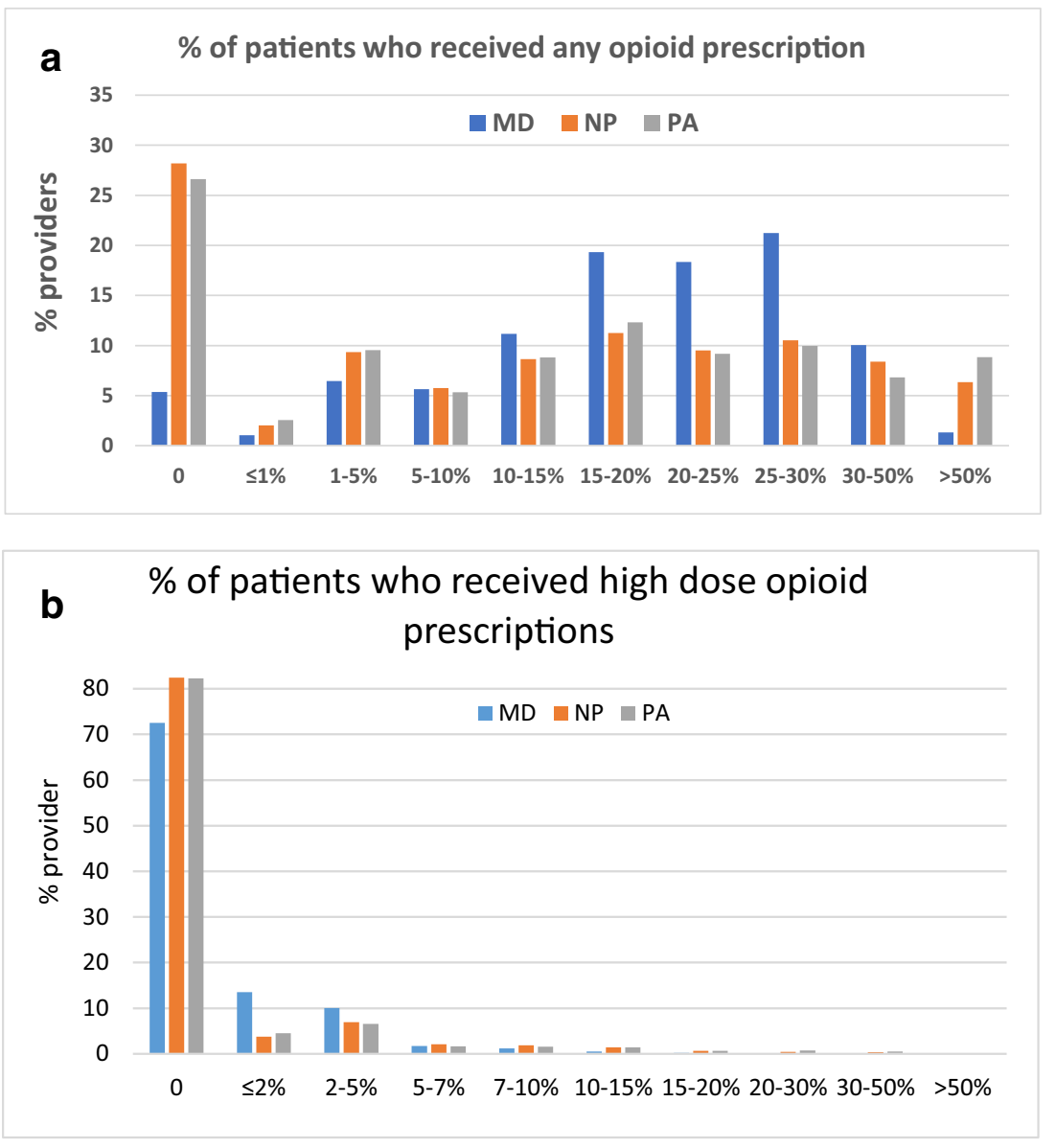

C $\quad \%$ of patients who received long-term opioid prescriptions

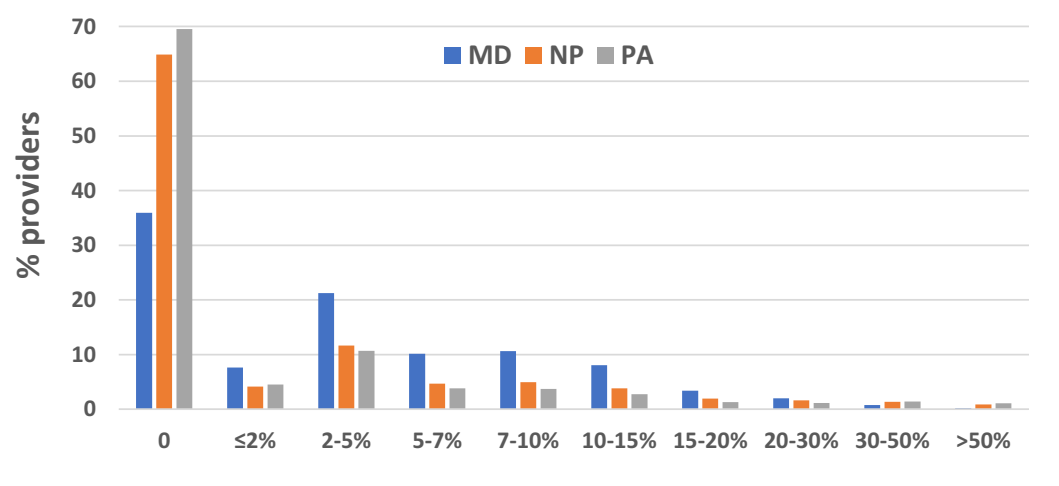

Fig. 1 Opioid prescribing metrics of individual providers, grouped by primary care MDs, NPs, and PAs. a Percent of patients receiving any opioid prescriptions in 2015, by provider. b Percent of patients receiving $>100$ MME per day. c Percent of patients receiving $\geq 90$ days of opioid prescriptions. MD, medical doctor; NP, nurse practitioner; PA, physician assistant; MME, morphine milligram equivalents.

\section{Sensitivity Analyses of Potential Opioid Overprescribing by Primary Care MDs, NPs, and PAs}

About $80 \%$ of primary care providers $(127,985 \mathrm{MDs} ; 34,175$ NPs; 19,569 PAs) met selection criteria for analysis of patient comorbidities (Supplemental Table 6). After adjusting for patient comorbidity in the multivariable analyses, we found similar results with somewhat lower odd ratios.
Compared with MDs, NPs/PAs were more likely to prescribe high-frequency (OR: NP, 2.26; PA, 4.36) and high-dose opioids (OR: NP, 1.29; PA, 1.62), but less likely to prescribe long-term opioids (OR: NP, 0.51; PA, 0.60) (Supplemental Table 7).

For sensitivity analysis of primary care specialty classification, 31,147 MDs, 9175 NPs, and 4983 PAs met modularity criteria from social network analysis. A subcohort $(11,172$ 
Table 2 Characteristics Associated with Opioid Overprescribing Between Types of Provider Within the Same State, Estimated from Conditional Logistic Regression Models

\begin{tabular}{|c|c|c|c|c|c|c|}
\hline & \multicolumn{2}{|c|}{ High frequency } & \multicolumn{2}{|c|}{ High dose } & \multicolumn{2}{|c|}{ Long term } \\
\hline \multicolumn{7}{|l|}{ Type of provider } \\
\hline Primary care NP & 2.96 & $2.78-3.15$ & 1.66 & $1.52-1.80$ & 0.57 & $0.53-0.61$ \\
\hline Primary care PA & 5.73 & $5.35-6.13$ & 2.16 & $1.97-2.38$ & 0.71 & $0.65-0.77$ \\
\hline \multicolumn{7}{|c|}{ Patient characteristics (every $10 \%$ increase), all provider types } \\
\hline$\%$ Age older than 80 & 1.37 & $1.34-1.40$ & 1.27 & $1.23-1.31$ & 1.27 & $1.24-1.31$ \\
\hline$\%$ Original entitlement as disabled & 1.81 & $1.77-1.86$ & 2.20 & $2.14-2.27$ & 2.16 & $2.11-2.21$ \\
\hline$\%$ Medicaid eligible & 0.97 & $0.95-0.99$ & 0.78 & $0.76-0.80$ & 0.90 & $0.89-0.92$ \\
\hline$\%$ HMO enrollee & 0.96 & $0.94-0.97$ & 0.92 & $0.91-0.94$ & 0.96 & $0.94-0.97$ \\
\hline Rurality (1-unit increase) & 0.88 & $0.86-0.90$ & 0.85 & $0.83-0.88$ & 1.05 & $1.04-1.07$ \\
\hline PCP available per 10,000 & 1.01 & $1.00-1.01$ & 1.01 & $1.01-1.02$ & 0.98 & $0.97-0.98$ \\
\hline
\end{tabular}

MD, medical doctor; NP, nurse practitioner; PA, physician assistant; HMO, health maintenance organization; PCP, primary care physician

MDs, 3303 NPs, 1728 PAs) was further identified as practicing in primary care practices (Supplemental Table 4). When we examined only providers in primary care practices for opioid overprescribing, we identified just $0.74 \%$ of primary care MDs as high-frequency prescribers compared with $7.5 \%$ of NPs and $6.0 \%$ of PAs. A small percentage of primary care providers prescribed high-dose opioids to more than $10 \%$ of their patients (MD $0.52 \%$, NP 2.36\%, PA 2.95\%). The percentage who prescribed long-term opioids to $>20 \%$ of their patients was slightly higher among NPs and PAs (MD 1.82\%, NP $4.01 \%$, PA 3.59\%).

\section{DISCUSSION}

The current retrospective study of 2015 Medicare Part D enrollees showed that $3.8 \%$ of MDs, $8.0 \%$ of NPs, and $9.8 \%$ of PAs overprescribed opioids.

Most primary care providers had similar opioid prescribing patterns; however, more NPs/PAs than MDs were outliers who prescribed high-frequency (any opioid to $>50 \%$ of patients) or high-dose opioids ( $\geq 100 \mathrm{MME} /$ day to $>10 \%$ of patients). NPs/ PAs in states with independent prescription authority for schedule II opioids were more likely to overprescribe opioids than

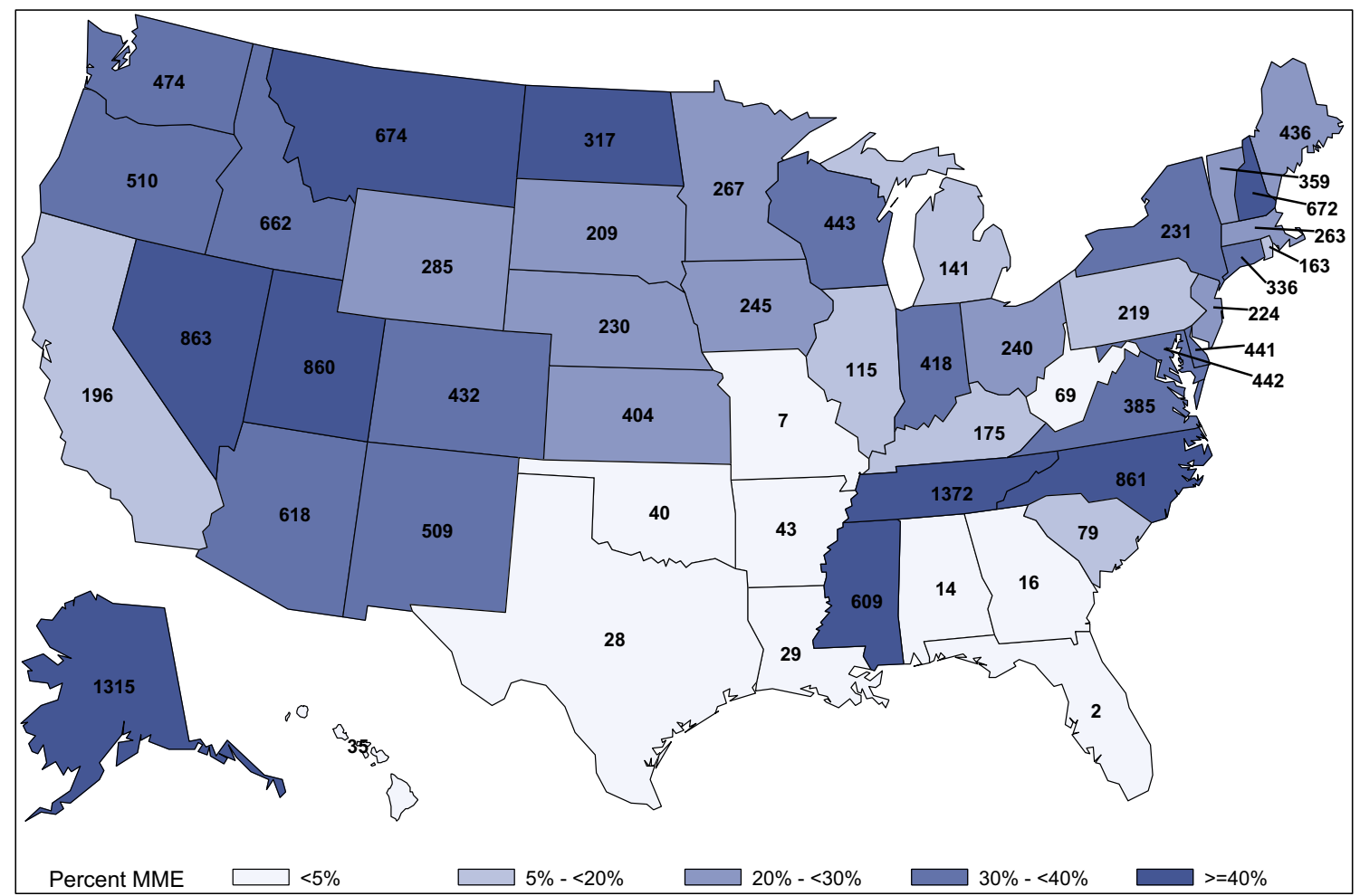

Fig. 2 Opioids prescribed to Medicare Part D enrollees by NPs/PAs. The MME prescribed by primary care NPs/PAs is displayed as a percentage of the total MME prescribed by all primary care providers (MDs, NPs, PAs) and ordered by quintile (shown in color). The number corresponding to each state shows the mean MME per Part D enrollee which primary care NPs/PAs in that state prescribed. MD, medical doctor; NP, nurse practitioner; PA, physician assistant; MME, morphine milligram equivalents. 
NPs/PAs in states with restricted prescription authority. All primary care providers were more likely to overprescribe opioids for Medicare enrollees aged $>80$, Whites, females, and disabled. Providers were less likely to overprescribe (or even prescribe) opioids for non-Whites, Medicaid, and rural residents. Opioid underprescribing to ethnic/racial minorities suggests potential under-treatment of pain, as indicated in past studies. ${ }^{28,} 29$

Emerging data indicates NPs/PAs as a group prescribe more opioids than MDs. ${ }^{30-32}$ Recent studies have found that generalist NPs/PAs prescribe a disproportionately high quantity of opioids to Medicare Part D beneficiaries compared with $\mathrm{MDs}^{30}$ and that NPs/PAs significantly increased opioid prescribing from 2013 to 2017, a period when almost every medical specialty decreased opioid prescribing. ${ }^{32}$ However, these studies lacked MME data and information about patient characteristics. Our study advanced prior research on NP/PA opioid prescribing by describing the frequency/potency/duration of prescriptions and by showing the effect of state policy on overprescribing. In states that granted independent prescription authority, $7.5 \%$ of NPs and $10.0 \%$ of PAs were high-frequency opioid prescribers. In states which restricted authority, only $0.18 \%$ of NPs and $0.74 \%$ of PAs were highfrequency prescribers. These findings differ from Ladd et al. ${ }^{33}$ who found state scope of practice legislation did not predict opioid prescribing in 2013. The discrepant findings likely reflect the fact that Ladd et al. grouped PAs and MDs together for their analysis. As our results show, opioid prescribing patterns of PAs are sensitive to prescription authority laws and are more closely aligned with NPs than MDs. Our findings support laws which limit non-physician prescribing of schedule II opioids.

Potential explanations for the high rate of opioid overprescribing by NPs/PAs in our study include the aggressive marketing of opioid products by pharmaceutical companies directly to NPs/PAs. ${ }^{30}$ Our study results may also reflect the possibility that NP/PA patients have a high burden of multiple painful conditions, requiring large numbers of opioid prescription refills. Alternatively, the practice setting of NPs/PAs may have contributed to higher rates of opioid prescribing, if, for example, acute pain was a common presenting complaint. Nonetheless, mandatory continued education (CE) in safe opioid prescribing should be a licensing requirement for all prescribers, especially NPs and PAs whose licensures and professional certifications do not require mandatory postprofessional degree residency training. Laws which aim to limit overprescribing (e.g., limits on pills prescribed, prescription drug monitoring database checking, and physician collaboration requirement) should remain in place. Our findings of high NP/PA overprescribing in states with independent prescription authority suggest that restricting NPs/PAs prescribing authority might reduce opioid overprescribing. Another approach to reducing overprescribing, especially among the outliers, is incorporating a system into the electronic health records that limits the number of opioid pills and total MME for patients being discharged from the hospital or ER. ${ }^{34}$

\section{Limitations}

Limitations include analysis of only 2015 Medicare claims data, a time near the peak of opioid prescribing in the USA. The dataset does not include the indication for prescriptions, which limits our ability to assess overprescribing appropriateness. Opioid prescribing for palliative care could confound results if, for example, NPs or PAs had more or less of these patients than MDs. Hospice/palliative claims were not requested for the dataset. We identified primary care providers using NPI information and taxonomy codes in Medicare data; but misclassification could occur, given the flexibility of NPs/ PAs to change specialty without the requirement of additional post-graduate residency. We limited analysis of the $20 \%$ sample dataset to those providers having at least 50 Medicare prescriptions. This selection criteria led to exclusion of $26 \%$ of MDs, $48 \%$ of NPs, and $53 \%$ of PAs. Therefore, we cannot generalize our findings to those who prescribed less often. Furthermore, we excluded NPs/PAs with $>50 \%$ E\&M billing from ER services and those certified in non-primary care areas (3.1\% of NPs, $9.9 \%$ of PAs). The proportion of exclusions was comparable to published reports for NPs but was much lower for PAs. ${ }^{35,}{ }^{36}$ However, our sensitivity analyses on the subcohort of providers in primary care practices showed similar results.

Opioid overprescribing is inconsistently defined in previous literature. We defined opioid overprescribing based on the frequency, potency (MME/day), and length of prescriptions, while using definitions similar to the CDC. ${ }^{11}$ Our findings from Medicare patients are not generalizable to younger, privately insured patients. These results also do not discriminate between patients who received opioids from one type of provider and those who received opioids from both MDs and NPs/PAs. Future work will apply multilevel analysis to compare prescribing differences to individual patients by the most frequent prescriber. Finally, 2015 prescribing data may not apply to later years given the passage of new laws regulating opioid prescribing. ${ }^{37-39}$ Such laws will likely lead to decreased variability in opioid prescribing, but the effect on opioid-related morbidity and mortality is unclear.

\section{CONCLUSIONS}

In 2015, 12 million Medicare Part D beneficiaries received an average of five separate opioid prescriptions. ${ }^{40}$ Primary care NPs and PAs prescribed these opioids more often and at higher doses than did primary care MDs. Furthermore, $8.0 \%$ of NPs and $9.8 \%$ of PAs met at least one definition of opioid overprescribing compared with $3.8 \%$ of MDs. In states that allowed NPs/PAs to independently prescribe schedule II controlled substances, NPs/PAs were $>20$ times more likely to overprescribe opioids than in states with restricted prescription authority. Future research to identify providers at highest risk of overprescribing requires a rigorous examination providers' 
years in practice, their educational backgrounds (NP vs DNP), associated diagnoses and pain scores, and patient volume and levels of acuity.

Corresponding Author: M. James Lozada, DO; Department of AnesthesiologyVanderbilt University Medical Center, 1211 Medical Center Dr., 4202 VUH, Nashville, TN, USA (e-mail: james. lozada@vumc.org).

Funding Information HHS | Agency for Healthcare Research and Quality: Yong-Fang Kuo, R01-HSO2O642.

HHS | National Institutes of Health (NIH): Yong-Fang Kuo, RO1DA039192, P3OAG024832, UL1TROO1439.

\section{Compliance with Ethical Standards:}

The University of Texas Medical Branch Institutional Review Board approved the research.

Conflict of Interest: The authors declare that they do not have a conflict of interest.

\section{REFERENCES}

1. Guy GP Jr., Zhang K, Bohm MK, et al. Vital signs: changes in opioid prescribing in the United States, 2006-2015. MMWR Morb Mortal Wkly Rep. 2017;66:697-704.

2. U.S. Department of Health \& Human Services. Office of Inspector General. Opioid Use Decreased in Medicare Part D, While Medication Assisted Treatment Increased. In. https://oig.hhs.gov/oei/reports/oei02-19-00390.pdf2019.

3. Scholl L, Seth P, Kariisa M, Wilson N, Baldwin G. Drug and opioidinvolved overdose deaths - United States, 2013-2017. MMWR Morb Mortal Wkly Rep. 2018;67(5152):1419-1427.

4. Seth P, Rudd RA, Noonan RK, Haegerich TM. Quantifying the epidemic of prescription opioid overdose deaths. Am $J$ Public Health 2018;108(4):500-502

5. U.S. Department of Health \& Human Services. Office of the Secretary. Determination that a Public Health Emergency Exists. In. https://www. hhs.gov/sites/default/files/opioid\%20PHE\%20Declaration-no-sig. pdf2017.

6. Burton BN, Lin TC, Said ET, Gabriel RA. National trends and factors associated with inpatient mortality in adult patients with opioid overdose. Anesth Analg. 2018.

7. Busse JW, Wang $\mathbf{L}$, Kamaleldin $\mathbf{M}$, et al. Opioids for chronic noncancer pain: a systematic review and meta-analysis. JAMA. 2018;320(23):24482460.

8. Ashburn MA, Fleisher LA. Increasing evidence for the limited role of opioids to treat chronic noncancer pain. JAMA. 2018;320(23):24272428.

9. Bohnert ASB, Guy GP, Jr., Losby JL. Opioid prescribing in the United States before and after the Centers for Disease Control and Prevention's 2016 opioid guideline. Ann Intern Med. 2018;169(6):367-375.

10. Neuman MD, Bateman BT, Wunsch H. Inappropriate opioid prescription after surgery. Lancet. 2019;393(10180):1547-1557.

11. Dowell D HT, Chou R. CDC Guidelines for prescribing prescription opioids for chronic pain-United States, 2016. In. MMWR Recomm Rep 2016; 65:1-502016.

12. Goodwin JS, Kuo Y, Brown D, Juurlink D, Raji M. Association of chronic opioid use with presidential voting patterns in us counties in 2016. JAMA Network Open. 2018;1(2).

13. Kuo YF, Raji MA, Liaw V, Baillargeon J, Goodwin JS. Opioid prescriptions in older Medicare beneficiaries after the 2014 federa rescheduling of hydrocodone products. $J$ Am Geriatr Soc. 2018;66(5):945-953.
14. Axeen S. Trends in opioid use and prescribing in Medicare, 2006-2012. Health Serv Res. 2018;53(5):3309-3328.

15. Kuo YF, Loresto FL, Jr., Rounds LR, Goodwin JS. States with the least restrictive regulations experienced the largest increase in patients seen by nurse practitioners. Health Aff (Millwood). 2013:32(7): 1236-1243

16. Auerbach DI, Staiger Do, Buerhaus PI. Growing ranks of advanced practice clinicians - implications for the physician workforce. $N$ Engl $J$ Med. 2018;378(25):2358-2360.

17. Frost A, Hargraves, J. HCCI Brief: Trends in Primary Care Visits. https:// www.healthcostinstitute.org/research/publications/hcci-research/entry/trends-in-primary-care-visits: Health Care Cost Institute; November 15, 20182018.

18. von Elm E, Altman DG, Egger M, et al. The Strengthening the Reporting of Observational Studies in Epidemiology (STROBE) statement: guidelines for reporting observational studies. Int $J$ Surg. 2014;12(12):14951499.

19. U.S. Department of Agriculture. Rural-Urban Continuum Codes. https://www.ers.usda.gov/data-products/rural-urban-continuumcodes/. Published 2017. Updated October 16, 2017. Accessed November $3,2018$.

20. Health Resources and Services Administration. Area Health Resource Files, County Level Data. In. https://data.hrsa.gov/data/download.

21. Phillips SJ. 28th Annual APRN Legislative Update: advancements continue for APRN practice. Nurse Pract. 2016;41(1):21-48

22. Henry J. Kaiser Family Foundation. Physician Assistant Scope of Practice Laws as of July 24, 2015. https://www.kff.org/other/stateindicator/physician-assistant-scope-of-practice-laws/?currentTimeframe $=0$ \&sortModel $=\% 7 \mathrm{~B} \% 22$ colId $\% 22: \% 22$ Adaptable $\% 20$ Supervision $\% 20$ Requirements $\% 3 \mathrm{~F} \% 22, \% 22$ sort $\% 22: \% 22$ desc $\% 22 \% 7 \mathrm{D}$. Updated July 24, 2015. Accessed November 2, 2018.

23. PA Role in Opioid Treatment Programs. American Academy of PAs. https://www.aapa.org/wp-content/uploads/2017/01/PA_Role in Opioid_Treatment_Programs.pdf. Published 2018. Accessed July 29, 2019.

24. Sigurdsson MI, Helgadottir S, Long TE, et al. Association between preoperative opioid and benzodiazepine prescription patterns and mortality after noncardiac surgery. JAMA Surg. 2019:e191652.

25. Yang Z, Algesheimer R, Tessone CJ. A comparative analysis of community detection algorithms on artificial networks. Sci Rep. 2016;6:30750

26. Fortunato S, Barthelemy $\mathbf{M}$. Resolution limit in community detection. Proc Natl Acad Sci U S A. 2007:104(1):36-41.

27. Kuo YF, Raji MA, Lin YL, Ottenbacher ME, Jupiter D, Goodwin JS. Use of Medicare data to identify team-based primary care: is it possible? Med Care. 2019;57(11):905-912

28. Institute of Medicine (US) Committee on Understanding and Eliminating Racial and Ethnic Disparities in Health Care. Unequal Treatment: Confronting Racial and Ethnic Disparities in Health Care. Washington (DC): National Academies Press (US)2003.

29. Ly DP. Racial and Ethnic Disparities in the Evaluation and Management of Pain in the Outpatient Setting, 2006-2015. Pain Med. 2018

30. Ellenbogen MI, Segal JB. Differences in opioid prescribing among generalist physicians, nurse practitioners, and physician assistants. Pain Med. 2019.

31. Muench U, Spetz J, Jura M, Guo C, Thomas C, Perloff J. Opioidprescribing outcomes of Medicare beneficiaries managed by nurse practitioners and physicians. Med Care. 2019.

32. Romman AN, Hsu CM, Chou LN, et al. Opioid prescribing to Medicare Part D enrollees, 2013-2017: shifting responsibility to pain management providers. Pain Med. 2020.

33. Ladd E, Sweeney CF, Guarino A, Hoyt A. Opioid prescribing by nurse practitioners in Medicare Part D: impact of state scope of practice legislation. Med Care Res Rev. 2017:1077558717725604.

34. Lowenstein $\mathbf{M}$, Hossain E, Yang $\mathbf{W}$, et al. Impact of a state opioid prescribing limit and electronic medical record alert on opioid prescriptions: a difference-in-differences analysis. $J$ Gen Intern Med. 2019

35. American Association of Nurse Practitioners (AANP). Nurse Practitioners in Primary Care. https://www.aanp.org/advocacy/advocacy-resource/ position-statements/nurse-practitioners-in-primary-care. Published 2013. Accessed January 08, 2019

36. Coplan B, Cawley J, Stoehr J. Physician assistants in primary care: trends and characteristics. Ann Fam Med. 2013;11(1):75-79. 
37. Goodman DC, Mick SS, Bott D, et al. Primary care service areas: a new tool for the evaluation of primary care services. Health Serv Res. 2003;38(1 Pt 1):287-309.

38. Pons P, Latapy M. Computing communities in large networks using random walks. In Computer and Information Sciences - ISCIS 2005. ISCIS 2005. Lecture Notes in Computer Science, vol 3733. Springer, Berlin, Heidelberg. In.

39. Chejara P, Godfrey Ww. Comparative Analysis of Community Detection Algorithms. 2017 Conference on Information and Communication
Technology (CICT17). https://ieeexplore.ieee.org/stamp/stamp.jsp? $\mathrm{tp}=$ \&arnumber $=8340627$. Accessed 08/15/2019. In.

40. U.S. Department of Health and Human Services. Office of Inspector General. High Part D Spending on Opioids and Substantial Growth in Compounded Drugs Raise Concerns. In: HHS OIG Data Brief, ed2016.

Publisher's Note: Springer Nature remains neutral with regard to jurisdictional claims in published maps and institutional affiliations. 